Musées, Patrimoine et Culture scientifiques et techniques

$178 \mid 2018$

juillet-août 2018

\title{
Des sciences citoyennes pour le développement durable
}

Marie-Lise Sabrié et Raphaële Nisin et Marie-Ève Migueres

\section{OpenEdition \\ Journals}

Édition électronique

URL : http://journals.openedition.org/ocim/2676

DOI : $10.4000 /$ ocim.2676

ISSN : 2108-646X

Éditeur

OCIM

Édition imprimée

Date de publication : 1 juillet 2018

Pagination : 26-31

ISSN : 0994-1908

Référence électronique

Marie-Lise Sabrié et Raphaële Nisin et Marie-Ève Migueres, « Des sciences citoyennes pour le développement durable », La Lettre de I'OCIM [En ligne], 178 | 2018, mis en ligne le 01 juillet 2019, consulté le 07 septembre 2019. URL : http://journals.openedition.org/ocim/2676 ; DOI : 10.4000/ ocim. 2676

Ce document a été généré automatiquement le 7 septembre 2019

Tous droits réservés 


\title{
Des sciences citoyennes pour le développement durable
}

\author{
Marie-Lise Sabrié et Raphaële Nisin et Marie-Ève Migueres
}

Les 17 objectifs du développement durable de l'ONU

\section{(6) OBJECTIFS}
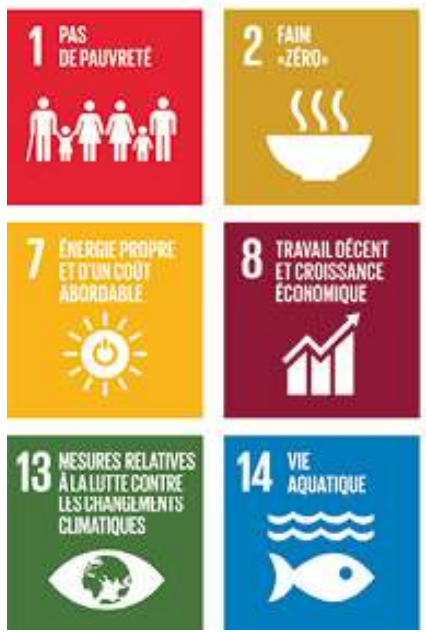
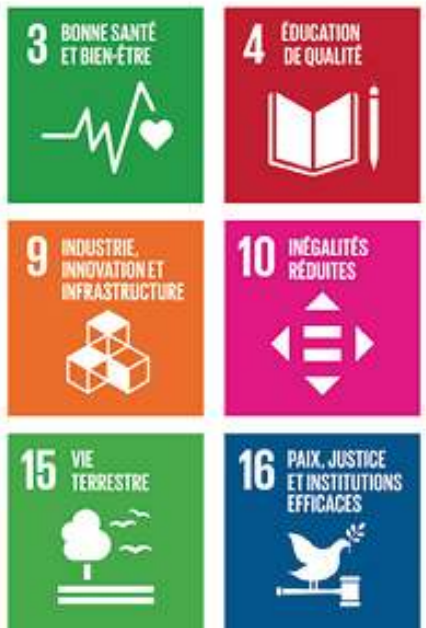
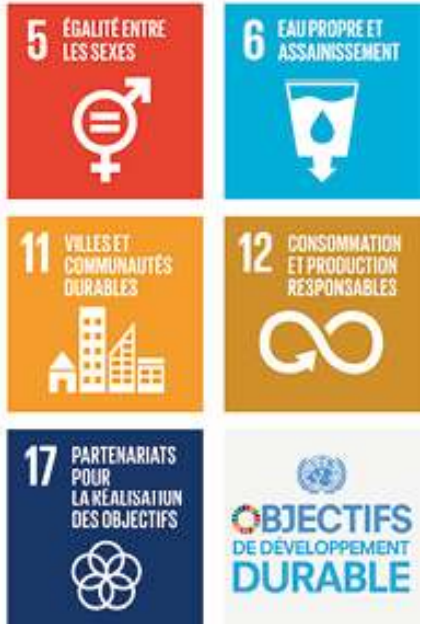

(86)

OBJECTIFS DEDEVELOPPEMENT

(c) DR

En 2015, lors d'un sommet historique des Nations-Unies, ses pays membres ont adopté un programme universel de développement durable qui vise en 17 objectifs à "transformer les vies tout en préservant la planète" d'ici à $2030^{1}$. Lutte contre la pauvreté, sécurité alimentaire, accès de tous à l'eau et à l'assainissement, modes de consommation, de production et d'industrialisation durables, préservation des écosystèmes terrestres et marins, mesures contre le changement climatique, construction de villes et de communautés durables... les 17 Objectifs du développement durable (ODD), aujourd'hui entrés en vigueur, invitent les États, les collectivités territoriales, les institutions 
internationales, les entreprises ou les organisations de la société civile à contribuer à un profond changement de modèle pour construire dans les décennies à venir, au Nord comme au Sud, des sociétés prospères et inclusives dans une planète préservée.

Parce qu'ils mobilisent les connaissances produites par les scientifiques dans les trois champs constitutifs du développement durable - économique, social, environnemental -, les ODD confèrent à la recherche, quelles que soient les disciplines concernées, des sciences de la Vie ou de la Terre aux sciences sociales, une place de premier plan. De fait, atteindre ces objectifs nécessite la production de données et d'analyses fiables pour établir de justes diagnostics, évaluer les objectifs et les actions engagées, concevoir des innovations adaptées et efficaces, mesurer les progrès enregistrés... "La science et les scientifiques sont dans une position clé pour faciliter la transformation de nos sociétés et de nos économies vers la durabilité. Ils peuvent contribuer à une bonne compréhension de la réalité et anticiper les effets des différentes combinaisons de mesures en même temps qu'ils conservent un regard critique et autonome sur ce qui se passe"2.

\section{La culture scientifique au service du développement durable}

3 Mais, pour que les données et les innovations de la recherche concourent effectivement aux ODD encore faut-il que celles-ci irriguent les programmes d'actions conçus et mis en œuvre par les instances politiques, entreprises ou organisations de la société civile. Encore faut-il aussi que les citoyens puissent s'approprier les questionnements, analyses et avancées de la recherche afin de s'affirmer comme des acteurs à part entière, compétents et informés, des politiques de développement durable. Comme le souligne Teresa Ribera, directrice de l'Institut du développement durable et des relations internationales (IDDRI), "il faut créer les conditions du dialogue avec les décideurs, et du dialogue avec la société. Une meilleure implication des citoyens dans la science (science citoyenne) permettrait une meilleure appropriation des résultats scientifiques, mais aussi une capacité plus vaste pour soutenir une action politique qui est nécessairement révolutionnaire si nous prenons au sérieux l'Agenda 2030"3.

Le renforcement de la capacité des citoyens à contribuer au développement durable est d'ailleurs inscrit au cœur de l'Agenda 2030 des Nations-Unies. L'objectif 4 fait ainsi de l'accès aux savoirs à travers une éducation de qualité, tout particulièrement à destination des jeunes, une clé de la participation citoyenne au développement durable : "D'ici à 2030, faire en sorte que tous les élèves acquièrent les connaissances et compétences nécessaires pour promouvoir le développement durable, notamment par l'éducation en faveur du développement et de modes de vie durables (...)"4. 
Une conférence débat lors de la COP 22 à Marrakech a réuni 150 lycées de France, d'Afrique de l'Ouest et du Maghreb pour dialoguer avec des décideurs sur le changement climatique.

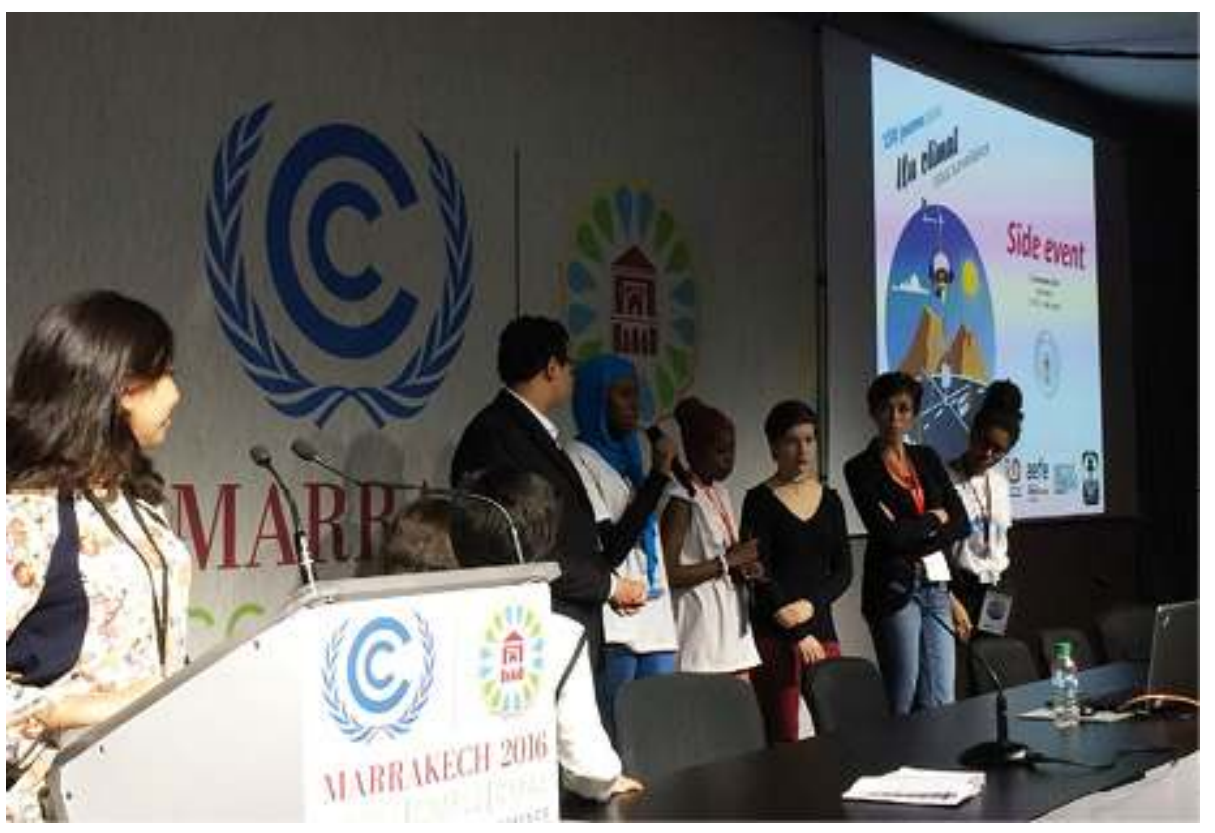

(C) IRD

De jeunes Sénégalaises interrogent les scientifiques de l'IRD dans le cadre du projet Climat sous Surveillance.

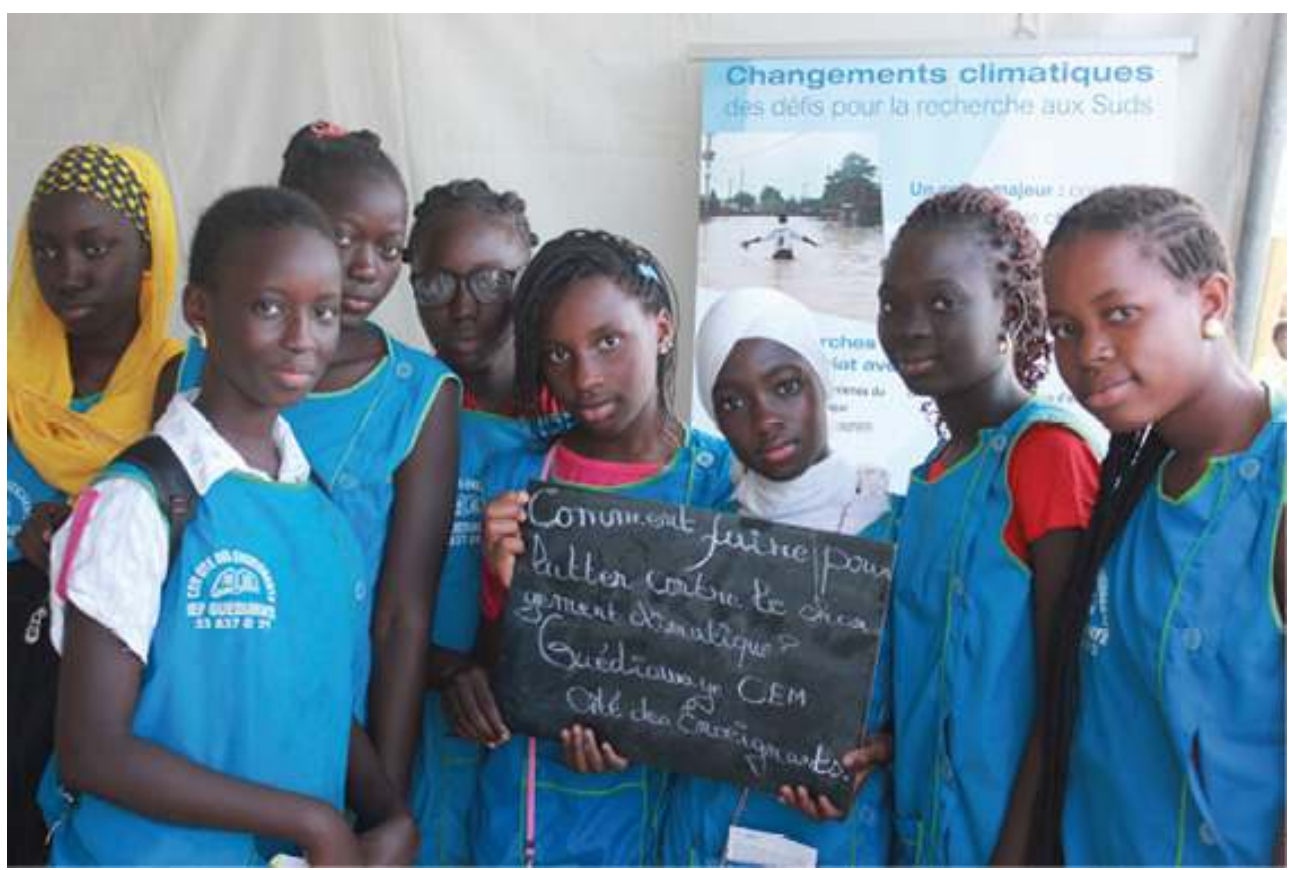

(c) IRD/DR

5 Comment favoriser une appropriation citoyenne des savoirs scientifiques pour atteindre les objectifs du développement durable ? En la matière, nul doute que les organismes de recherche comme l'IRD (Institut de recherche pour le développement) ont un rôle clé à jouer par les connaissances qu'ils produisent et qu'ils diffusent. L'IRD ${ }^{5}$, organisme public 
de recherche pluridisciplinaire dédié au développement par la coopération scientifique avec les pays du Sud, s'est fixé parmi les objectifs prioritaires de son plan d'orientation stratégique la mise en œuvre d'une politique de diffusion de la culture scientifique au service du développement durable : "Dans notre société dite "de la connaissance" où le savoir est le premier des biens communs, une recherche dont les avancées ne seraient pas largement diffusées et partagées avec les populations ne peut contribuer effectivement au développement, à la lutte contre les inégalités et à une plus grande justice sociale. La diffusion et le partage des savoirs issus de la recherche contribuent en effet à élever la capacité des citoyens à faire face aux multiples défis du développement, à effectuer des choix - personnels ou professionnels - éclairés et à être des acteurs à part entière du changement" ${ }^{16}$. Et ces actions de diffusion de la culture scientifique sont d'autant plus nécessaires que la fracture entre sciences et sociétés dans les pays en développement où intervient l'IRD est encore plus marquée qu'ailleurs, avec, comme au Nord, une forte désaffection des jeunes générations pour les enseignements et les filières scientifiques ${ }^{7}$. Les liens entre le monde de la recherche et la société sont d'autant plus ténus que, dans nombre de pays du Sud, en particulier en Afrique subsaharienne, rares sont les institutions dédiées à une médiation et à une "mise en culture" des savoirs scientifiques : musées, muséums, centres culturels, médias ou associations... Or, ces structures, aussi différentes soient-elles, peuvent être amenées à jouer un rôle majeur pour créer les conditions d'une meilleure appropriation par la société des connaissances et des innovations issues de la recherche.

\section{Citoyenneté et recherche en synergie}

6 Dans de multiples domaines qui relèvent des champs prioritaires définis par les 17 ODD, l'IRD développe des programmes d'actions qui visent à favoriser une appropriation des avancées de la recherche par les citoyens - en particulier les jeunes -, à les initier à la démarche scientifique et à favoriser leurs échanges avec la communauté scientifique. Parmi les actions mise en œuvre par l'IRD, deux projets - Villes en questions et ePOP (Petites Ondes participatives) - illustrent bien comment une politique de diffusion de la culture scientifique portée par un organisme de recherche peut contribuer à la construction d'une citoyenneté informée, active et engagée dans le développement durable.

7 Le onzième des 17 objectifs promus par l'ONU vise à l'horizon 2030 à la constitution de villes et de communautés durables. Dans cette perspective, le projet Villes en question invite des jeunes Méditerranéens (Algérie, France, Maroc, Tunisie) à poser un regard éclairé sur les grands défis urbains contemporains en les incitant à décrypter les liens sociaux dans leur ville ${ }^{8}$. Au cœur de ce projet, un double objectif : comment sensibiliser les jeunes à la question des villes durables ? Comment les accompagner pour qu'ils deviennent des acteurs qui participent de manière compétente et informée au devenir de leur ville? 
Projet Villes en Questions : enquête de terrain des lycéens de Beni Mellal au Maroc, encadrés par un anthropologue de l'IRD.

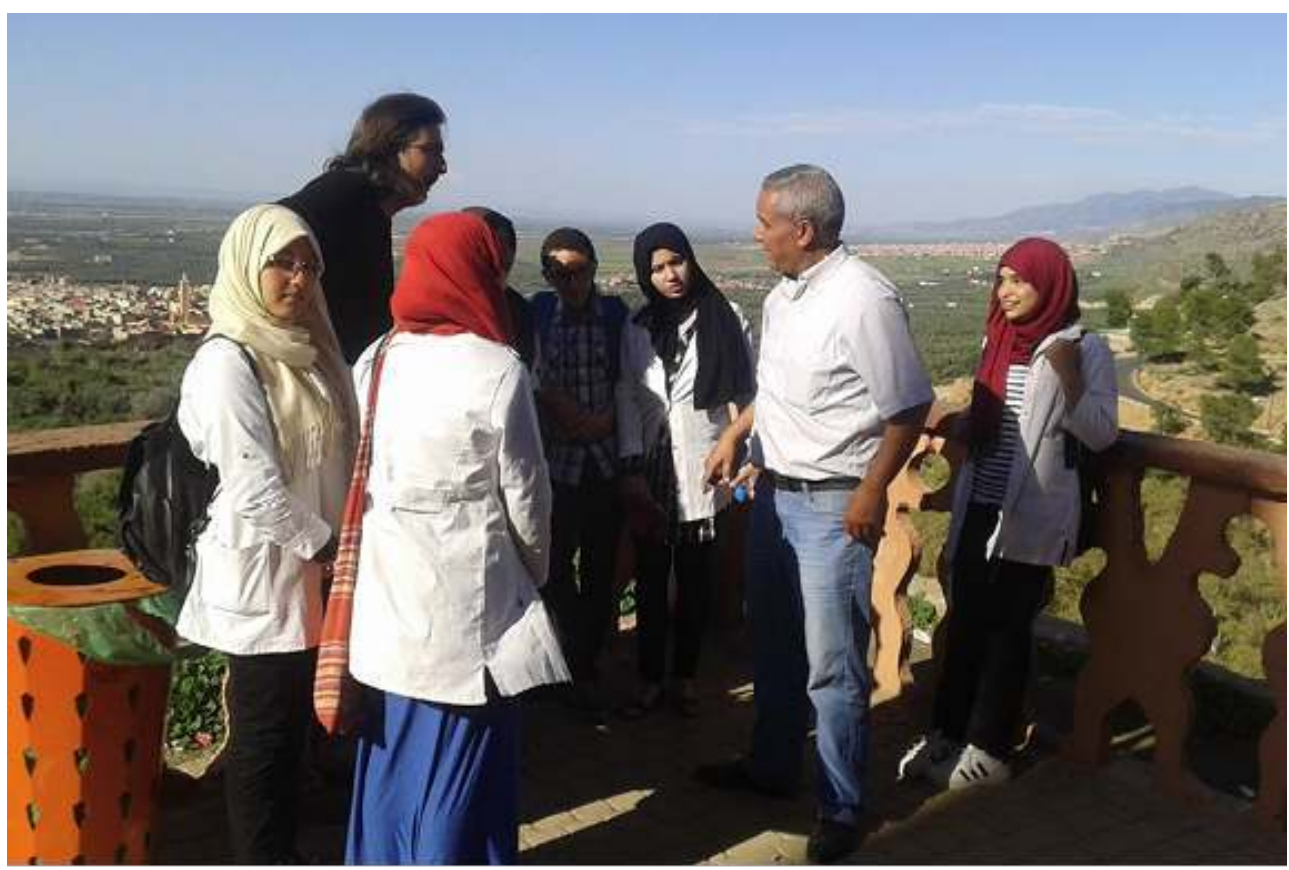

(c) AESVT

8 Accompagnés par des associations de médiation ${ }^{9}$ et leurs enseignants, conseillés par des chercheurs en sciences sociales (géographes, historiens, sociologues, économistes, anthropologues), les lycéens mènent des enquêtes dans leurs quartiers sur des sujets qui font l'actualité : migrations, transports, aménagements, alimentation, arts urbains ou accès à l'eau. Comparaison du développement urbain du centre ville et du nouveau quartier d'affaires Euroméditerranée à Marseille, réappropriation de la ville d'Alger par la culture, accueil des communautés Roms dans la commune de Gardanne, conflits entre urbanisation et activité agricole dans la banlieue de Béni Mellal au Maroc, ou encore les usagers du train de banlieue Tunis-Goulette-Marsa, offrent quelques exemples des thèmes d'enquête choisis par le millier de lycéens mobilisés par le dispositif depuis 2011. Le choix d'un thème d'enquête par les élèves - démarche privilégiée par le projet - ne fait pas toujours évidence notamment en raison de difficultés pour ces derniers à problématiser les sujets et peut obliger les encadrants à imposer une thématique, avec le risque d'une moindre adhésion des jeunes à l'exercice.

9 Apprentis chercheurs le temps d'une année, "les élèves sont amenés à développer une démarche scientifique rigoureuse", souligne Claude de Miras, chercheur émérite à l'IRD et parrain du projet ${ }^{10}$. Ils problématisent le sujet qu'ils ont choisi, observent, recueillent des données, analysent les résultats et proposent des conclusions. Les enquêtes de terrain peuvent parfois susciter l'appréhension des élèves qui redoutent d'avoir à questionner "l'autre", nécessitant des sorties encadrées pour les aider à surmonter ces réticences.

10 Cet apprentissage actif de la méthode d'enquête des sciences sociales leur permet de construire leurs propres connaissances. Dès lors, ils sont à même d'analyser avec plus de recul et moins d'idées reçues le monde urbain qui les entoure. "Suite aux sorties sur le terrain, les élèves ont développé de nouvelles compétences, la démarche utilisée leur a permis de prendre confiance en eux et de porter un regard distancié sur leur sujet d'étude", souligne ainsi 
un enseignant. "Ces entretiens les ont secoués, ils ont commencé à contourner leurs préjugés", ajoute pour sa part un médiateur.

11 À l'issue de leur année d'investigation, plus de 200 jeunes des différents pays sont invités à participer à un colloque de restitution de leurs travaux. À cette occasion, ils échangent sur leurs enquêtes respectives et dialoguent avec les experts. Certains groupes, comme celui du lycée de Khemisset au Maroc, ont même été rendre compte à la municipalité des résultats de leur enquête sur les jardins publics, lui proposant des recommandations, laquelle en retour s'est engagée à en tenir compte dans de futurs aménagements. "Sur l'ensemble des élèves, plus de $90 \%$ affirment avoir développé un regard nouveau sur leur ville, et disent vouloir agir pour elle suite à ce projet", indiquent les pilotes du dispositif. Au-delà de ses enjeux immédiats, le projet favorise aussi l'ouverture des jeunes vers d'autres citoyens de la Méditerranée. "Le temps du programme Villes en questions, la mise en synergie de la recherche et de la citoyenneté a permis de dépasser les clivages sociaux inhérents au bassin méditerranéen", se félicite Claude de Miras.

\section{Un réseau citoyen d'information et d'échanges}

12 Le treizième des objectifs de développement durable concerne les mesures de lutte contre le changement climatique et ses impacts, avec l'éducation parmi celles préconisées de manière prioritaire : "Améliorer l'éducation, la sensibilisation et les capacités individuelles et institutionnelles en ce qui concerne l'adaptation aux changements climatiques, l'atténuation de leurs effets et la réduction de leur impact". Depuis plusieurs années, l'IRD a développé des projets de sensibilisation des populations des pays du Sud, particulièrement vulnérables à ces bouleversements du climat ${ }^{11}$.

13 Parmi ces actions, le projet ePOP, lancé en 2016 par RFI Planète Radio et l'IRD dans les territoires du Pacifique, qui subissent de plein fouet les effets des changements climatiques et environnementaux majeurs. Dans plusieurs îles océaniennes (NouvelleCalédonie, Vanuatu, Fidji, Salomon, Nouvelle-Zélande notamment), ce réseau citoyen d'observation, d'information et d'échanges mobilise des lycéens ou des étudiants de premier cycle universitaire. À la suite de séances de formation aux techniques de prises de vue vidéo, ces jeunes recueillent avec leur smartphone, le ressenti et le questionnement des populations qui les entourent (notamment les anciennes générations) face au changement climatique. Ces témoignages sont ensuite portés auprès de scientifiques qui y apportent leur expertise et analyse. Constituant des capsules vidéos de quelques minutes, ces témoignages des insulaires et les réponses des scientifiques sont largement diffusés via plusieurs canaux - médias locaux, plateforme Internet du projet, réseaux sociaux, chaînes du groupe France Média Monde (RFI, France 24) - qui offrent une très large audience aux paroles recueillies. Si le principe du projet séduit la communauté scientifique, la courte durée des séquences vidéo, imposée par les modalités de diffusion, peut parfois susciter les réticences de chercheurs peu habitués à présenter leurs analyses en un temps très restreint. 
Deux jeunes Calédoniens du réseau ePOP interviewent un ancien sur les impacts du changement climatique.

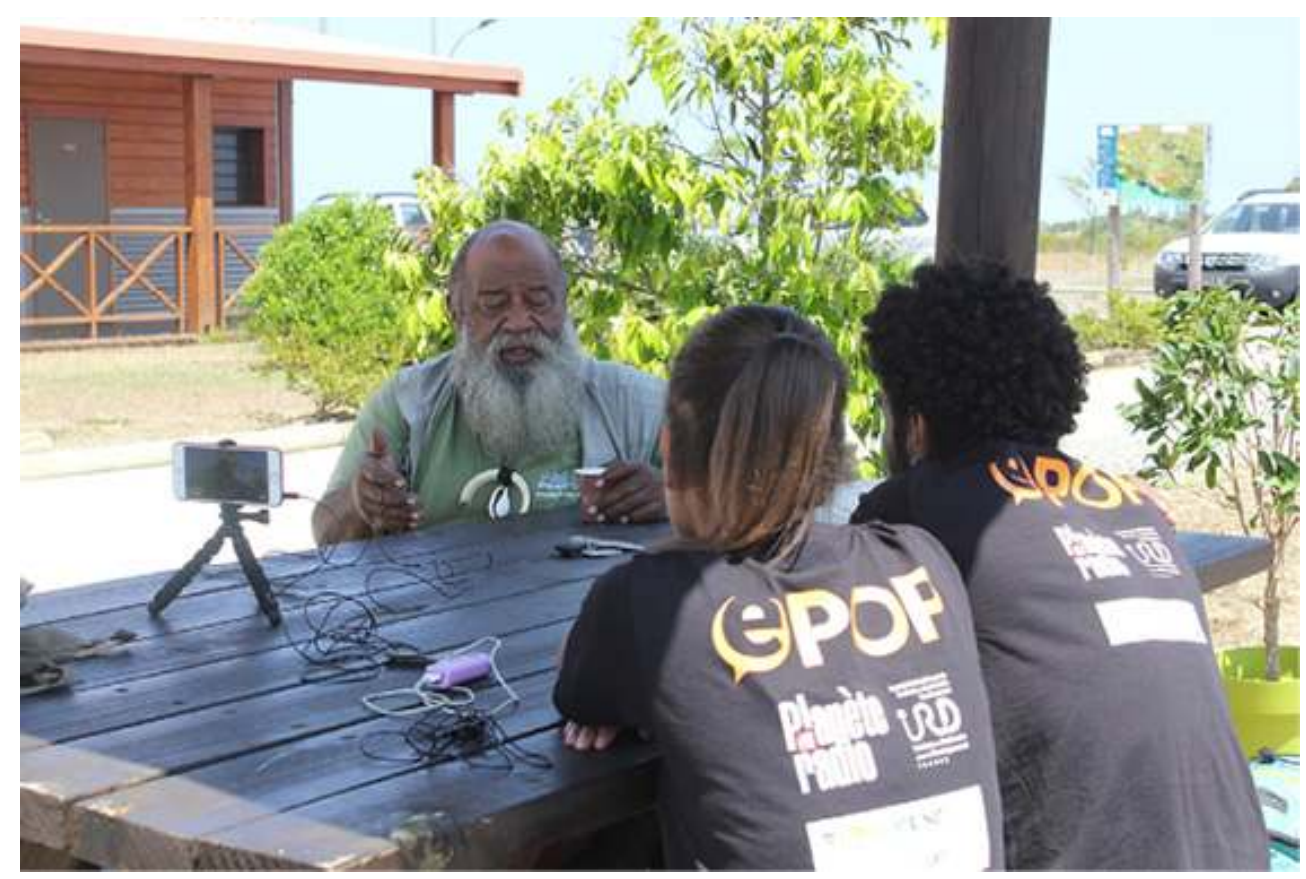

(C) IRD

Un étudiant fidjien réalise à l'aide d'un simple smartphone une vidéo sur les changements climatiques dans son île, qui sera très largement diffusé dans les médias régionaux et internationaux.

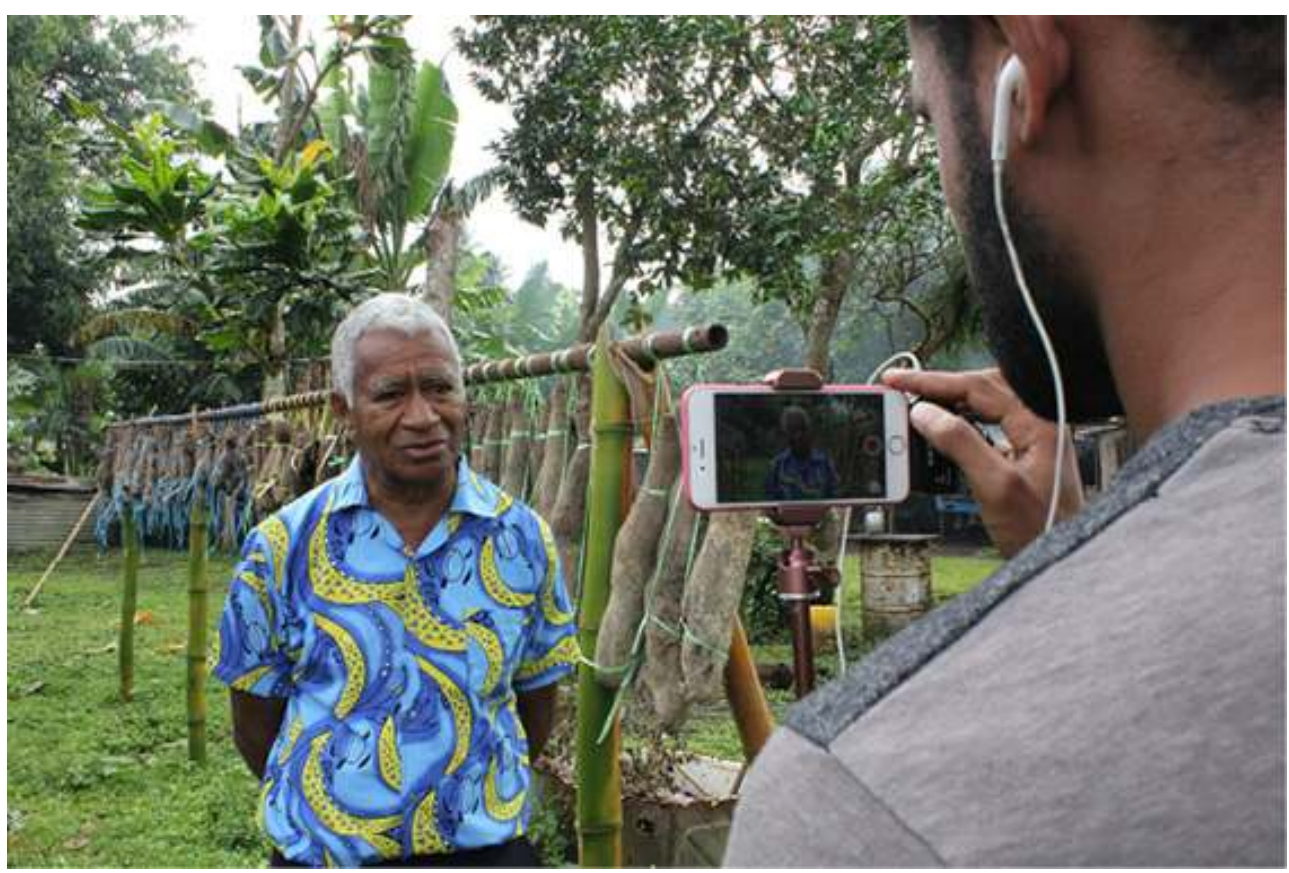

(C) IRD

14 Ce projet a pour premier intérêt de confier un rôle actif à la jeunesse insulaire en lui offrant l'opportunité de s'investir comme porte-parole des communautés qui les entourent. "ePOP est une opportunité extraordinaire de pouvoir rencontrer des journalistes de 
renom et un rêve de pouvoir exprimer nos craintes et inquiétudes sur des chaînes reconnues sur le plan international! Une chance pour nous les jeunes océaniens de pouvoir porter la voix de nos populations", souligne ainsi Medjo, étudiante de Nouvelle-Calédonie. Pour l'un des enseignants de l'école de journalisme de l'université du Pacifique Sud associée au projet, $e P O P$, outre la diffusion de connaissances scientifiques, permet également de "créer des conteurs multimédias du Pacifique responsables et compétents". "Avec les outils et la formation appropriés, précise-t-il, [les jeunes] acquièrent la capacité de raconter ces histoires comme aucune équipe de production étrangère ne pourrait le faire. Cela vient d'une compréhension profonde de leur propre culture et de leurs modes de narration".

Les jeunes du projet $e P O P$ sont également conviés à porter les témoignages recueillis auprès d'instances politiques, de représentants d'institutions scientifiques ou économiques en participant à des sommets internationaux. C'est ainsi que trois jeunes représentants du réseau se sont portés volontaires pour intervenir lors de la COP 23 sur les changements climatiques (Bonn, novembre 2017) pour interpeller le ministre fidjien de l'environnement, des représentants de la Banque Mondiale, du World Ressources Institute (think thank américain) et de l'université du Pacifique Sud, ou encore le directeur du Pacific Islands Development Forum, structure régionale en faveur d'une économie verte. Mobilisant les instances politiques ou scientifiques, ePOP joue ainsi un rôle de catalyseur et de caisse de résonnance de la parole des insulaires, dont la voix est rarement entendue dans de telles instances. "Les mondes scientifiques, économiques et politiques qui n'avaient pas d'outils pour entendre les témoignages des populations insulaires pour analyser les changements climatiques pourront désormais s'appuyer sur ePOP", souligne ainsi un étudiant des îles Salomon.

Pour la marraine du projet, Élisabeth Holland, directrice du Pacific Center for Environment and Sustainable Development et co-récipendiaire du prix Nobel de la Paix en tant que membre $d u$ GIEC, nul doute que ePOP fait des jeunes des acteurs à part entière du développement durable : "l'initiative est particulièrement utile dans le plaidoyer et le partage de l'information par l'entremise de nos étudiants et de nos communautés à travers le Pacifique, sur le changement climatique et le développement durable". 
Lors de la COP 23 à Bonn, des ePOPers interpellent des représentants d'Institutions internationales sur leurs actions dans la lutte contre le changement climatique.

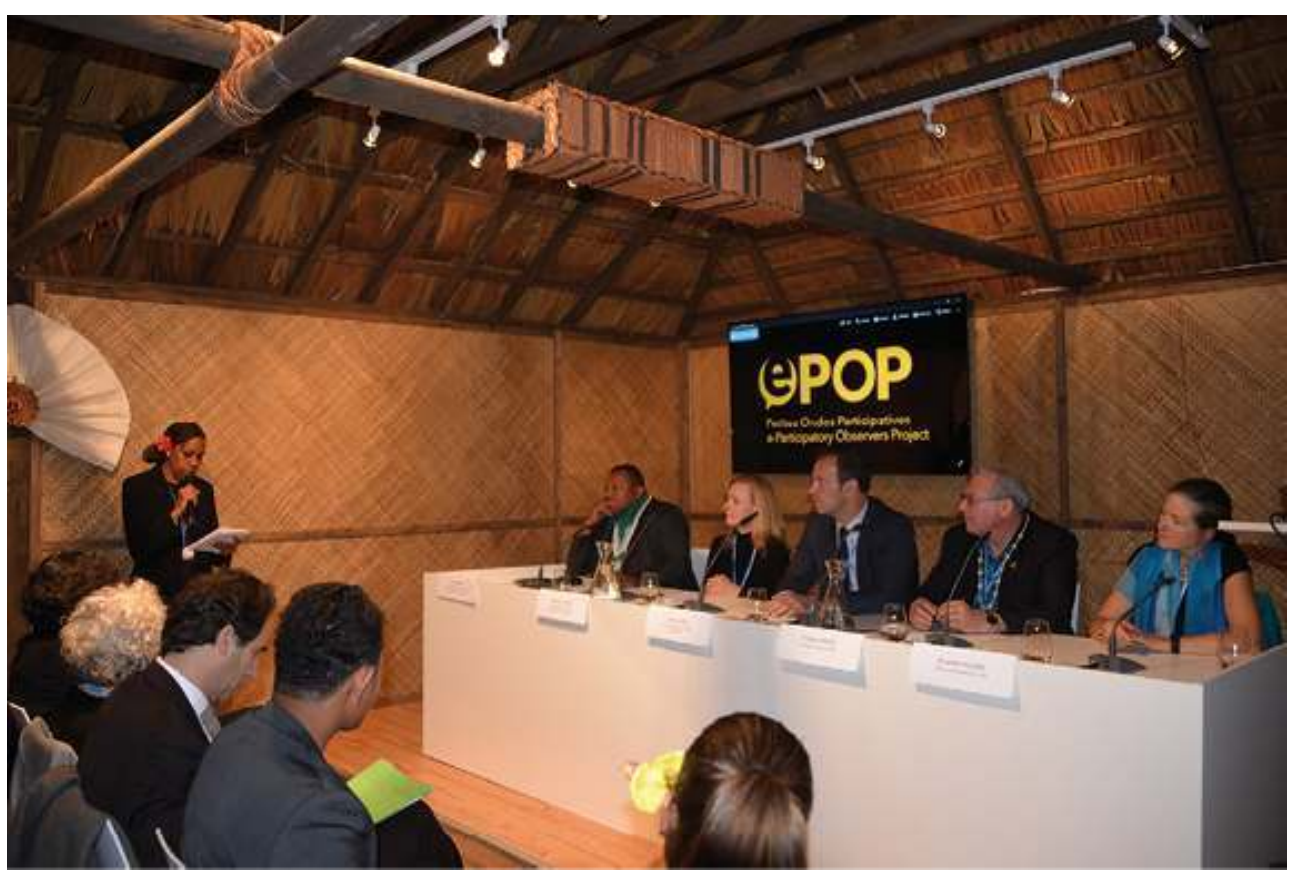

(C) RFI

Les actions mises en œuvre dans le Pacifique ces deux dernières années constituent la phase pilote de $e P O P$. Les résultats encourageants obtenus amènent aujourd'hui l'IRD et RFI Planète Radio à envisager son développement sur d'autres rivages, également menacés par le changement climatique : ceux de l'océan Indien ou d'Afrique de l'Ouest.

$\mathrm{Si}$, comme le montrent ces deux exemples, les actions de diffusion de culture scientifique portées par un institut de recherche renforcent la capacité des citoyens à devenir des acteurs à part entière du développement durable, celles-ci ont aussi comme vertu des créer des échanges entre une communauté scientifique et la société. Ces échanges ne sont pas aux seuls bénéfices des citoyens, ils sont aussi essentiels aux chercheurs auxquels ils permettent de mieux cerner les questionnements et interrogations des citoyens face au monde qui les entoure, d'appréhender leurs attentes vis-à-vis de la science, et de mieux en tenir compte dans l'élaboration de leur programme de recherche. Ce faisant, ce dialogue avec la science inscrit la recherche scientifique de plain-pied dans les objectifs du développement durable. 


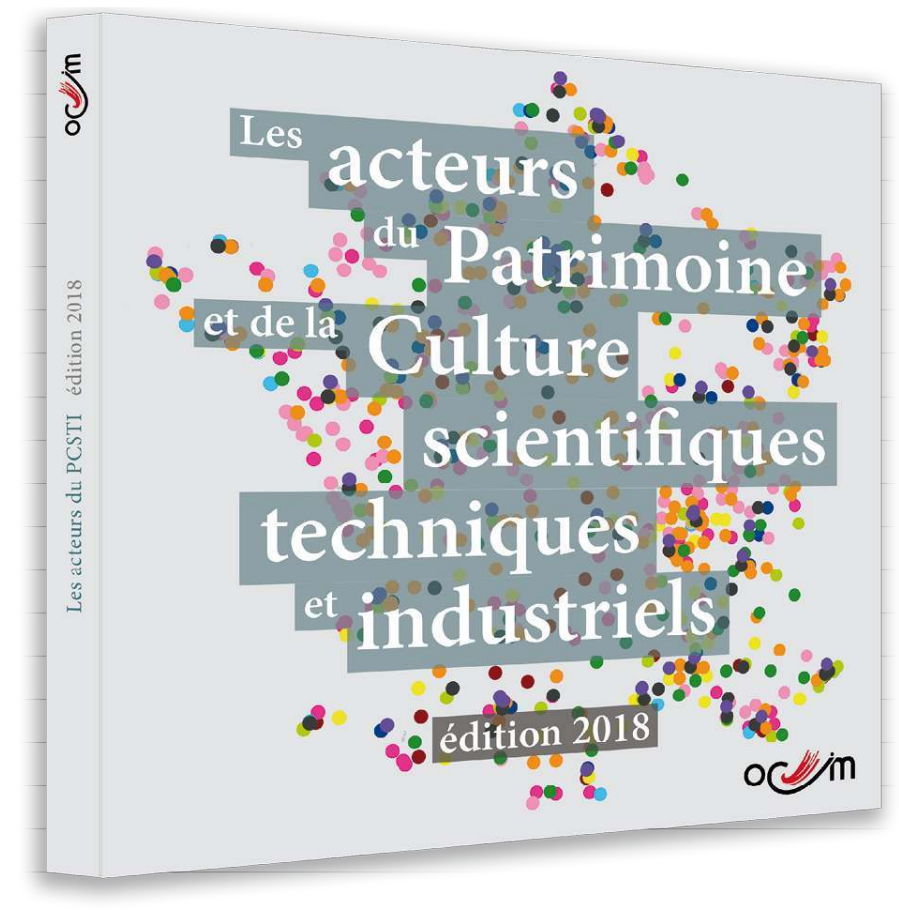

Cette édition 2018 constitue un instantané de la cartographie interactive correspondante disponible sur : www.ocim.fr. Tout comme sa version en ligne, elle offre des modalités de consultation des 1007 acteurs recensés par familles et par régions administratives. Ainsi, cette publication valorise l'inventaire des acteurs réalisé par l'Ocim dans le cadre de sa mission nationale d'observation du champ du PCSTI.

Le livret Les acteurs du Patrimoine et de la Culture scientifiques, techniques et industriels est téléchargeable gratuitement sur www.ocim.fr et disponible en version reliée au prix de $15 €$ ttc.

\section{NOTES}

1. www.un.org/sustainabledevelopment/fr/objectifs-de-developpement-durable/

2. Ribera, T. L'Agenda 2030 : un dialogue nécessaire entre science, société et politique, in Un Défi pour la planète - Les Objectifs du développement durable en débat, chapitre 4, Éditions Quae et IRD Éditions, 2017.

3. Teresa Ribera, ibid.

4. www.globalgoals.org/fr/global-goals/quality-education

5. www.ird.fr

6. IRD Plan d'orientation stratégique 2016-2030, pp. 21-22, en ligne sur www.ird.fr

7. Sabrié, M.-L. Promouvoir la culture scientifique en Afrique, La Lettre de l'Ocim, $\mathrm{n}^{\circ} 128$, marsavril 2010, pp. 5-11.

8. www.jeunes.ird.fr/villes-en-questions 
9. AMSSA : Association méditerranéenne de sciences sociales appliquée, Marseille; AESVT : Association des enseignants de sciences de la vie et de la terre, Maroc

10. Sciences au Sud, $n^{\circ} 83$, juin-octobre 2016

11. Climat sous surveillance : www.climat-sous-surveillance.ird.fr ; e-Pop : https:// epop.network/

\section{RÉSUMÉS}

Un programme universel de développement durable a été récemment lancé par l'ONU. Dans quelle mesure la diffusion de la culture scientifique participe-t-elle à sa mise en œuvre ? Des projets pédagogiques innovants portés par l'Institut de recherche pour le développement (IRD) montrent combien une meilleure appropriation des avancées des sciences peut contribuer à la construction d'une citoyenneté informée, active et engagée dans le développement durable.

\section{INDEX}

Mots-clés : Société, inclusif, expérience

\section{AUTEURS}

\section{MARIE-LISE SABRIÉ}

Marie-Lise Sabrié est directrice de la Mission Culture scientifique et technologique, IRD marie-lise.sabrie@ird.fr Raphaële Nisin est responsable adjointe du service Médiation avec les publics, IRD raphaele.nisin@ird.fr Marie-Ève Migueres est chargée de projet Médiations avec les publics, IRD marie-eve.migueres@ird.fr 